\title{
Upgrade of the STAR silicon detectors
}

\author{
Michal Szelezniak ${ }^{1}$ for the STAR collaboration \\ Institut Pluridisciplinaire Hubert CURIEN (IPHC) \\ 23 rue du Loess - BP28, 67037 Strasbourg, France \\ E-mail: michal.szelezniakeiphc.cnrs. fr
}

\begin{abstract}
Heavy Flavor Tracker (HFT) was installed in the center of the Solenoidal Tracker At RHIC (STAR) experiment for the 2014 heavy ion run of the Relativistic Heavy Ion Collider (RHIC). The HFT has been designed and built to extend the measurement capabilities of STAR in the heavy flavor domain by significantly improving its vertex resolution. The HFT consists of three silicon detectors arranged in four concentric cylinders around the STAR interaction point. The outermost layer, called Silicon Strip Detector (SSD), is located at the radius of $22 \mathrm{~cm}$ and is based on double-sided silicon strips. The second HFT layer, located at $14 \mathrm{~cm}$, is the Intermediate Silicon Tracker (IST) based on the silicon pad technology. The two innermost layers at 8 and $2.8 \mathrm{~cm}$ radii are provided by the PXL detector, which is based on state-of-the-art CMOS monolithic active pixel sensors (MAPS) technology featured for the first time in an accelerator experiment. The excellent pointing resolution of the HFT is driven by the exceptional characteristics of the PXL, which include high granularity and low material budget that limits the Multiple Coulomb Scattering in the detector layers. The very high granularity provided by the $20.7 \mu \mathrm{m}$ pixel pitch is combined with a matching mechanical stability. The low material budget of $0.4 \%$ radiation length per layer was achieved by using sensors thinned to $50 \mu \mathrm{m}$ and their low power dissipation that allows for simple air-cooling. A novel mechanical approach to detector insertion enables effective installation and integration of the PXL detector within a 12 hour shift during the on-going STAR Run. In this paper we will describe the HFT detector with a particular focus on the PXL detector design and implementation followed by the detector status during the $2014200 \mathrm{GeV} \mathrm{Au}+\mathrm{Au}$ run. We will report on a preliminary estimate of the HFT performance that meets the design requirements and summarize experience from the construction and operation of the PXL detector.
\end{abstract}

The 23rd International Workshop on Vertex Detectors

15-19 September 2014

Macha Lake, The Czech Republic

${ }_{1}^{1}$ Speaker
๑ Copyright owned by the author(s) under the terms of the Creative Commons Attribution-NonCommercial-ShareAlike Licence. 


\section{Introduction}

Results obtained in experiments at the Relativistic Heavy Ion Collider (RHIC) over the last decade suggest that a hot and dense matter with strong collectivity has been formed in central $\mathrm{Au}+\mathrm{Au}$ collisions. This indicates the existence of a phase of deconfined quarks and gluons, the so-called Quark-Gluon Plasma (QGP) [1]. Due to their mass, heavy quarks are only produced by hard processes early in the collision and not by thermal processes after the equilibration of the plasma. This property makes mesons containing heavy quarks an ideal probe for studying the initial conditions of the produced QGP. Most of the heavy quarks produced in the heavy ion collisions end up in open heavy flavor particles that until recently were hard to detect in the STAR experiment due to their low abundance and the large combinatorial background. To extend the STAR measurement capabilities in the heavy flavor domain, a new vertex detector called Heavy Flavor Tracker (HFT) has been installed in STAR before the beginning of the 2014 heavy ion RHIC run. The HFT provides an excellent vertex pointing capability that enables measurements of decay vertices of open heavy flavor particles with very short lifetimes (such as the $\mathrm{D}^{0}$ meson with $\mathrm{c} \tau \sim 100 \mu \mathrm{m}$ ) by direct topological reconstruction [2].

The HFT is installed inside the inner field cage of the STAR Time Projection Chamber (TPC, the main tracking detector in STAR) and consists of three silicon sub-detectors arranged in four cylindrical layers around the collision point as shown in Figure 1. The outermost layer based on silicon strip technology (Silicon Strip Detector, SSD) is located at $22 \mathrm{~cm}$ from the beam line. The Intermediate Silicon Tracker (IST) features single-sided silicon pad sensors located at $14 \mathrm{~cm}$ radius. The two innermost layers at 8 and $2.8 \mathrm{~cm}$ radii constitute the PXL detector that is based on state-of-the-art CMOS Monolithic Active Pixel Sensors (MAPS) [3]. Equipped with the HFT, the STAR detector is expected to provide the distance of closest approach (DCA) pointing resolution of $\leq 60 \mu \mathrm{m}$ for $750 \mathrm{MeV} / \mathrm{c}$ kaons. This resolution is achieved by tracking inwards from the TPC, with pointing resolution of approximately $1 \mathrm{~mm}$, through the SSD and IST with pointing resolutions of 250-300 $\mu \mathrm{m}$ to the PXL detector that can point at the secondary vertices with the resolution of a few tens of micrometers.

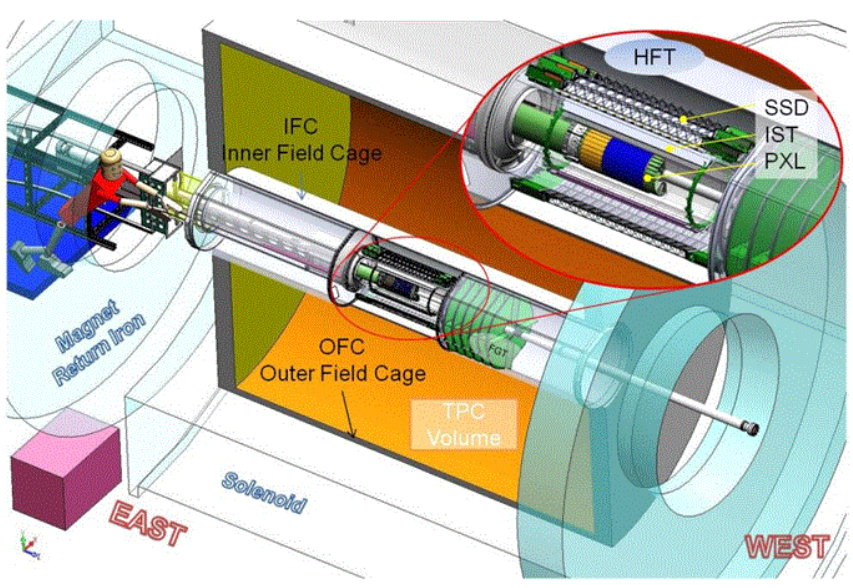

A

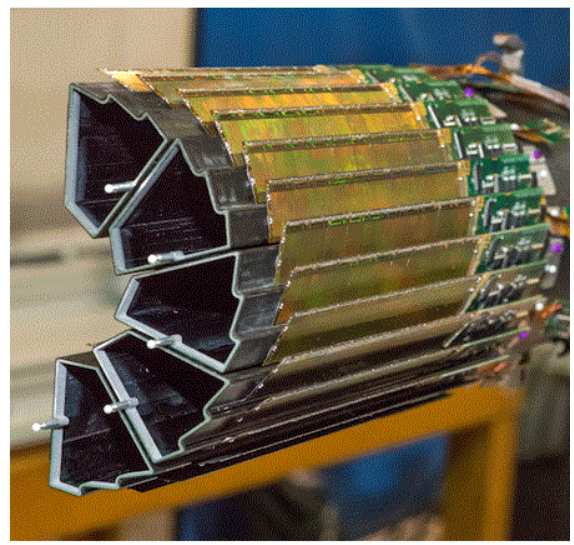

$\mathrm{B}$

Figure 1 A - structure of the STAR HFT upgrade with SSD, IST and PXL at 22, 14 and $8,2.8 \mathrm{~cm}$ radii, respectively $B$ - Image of the cantilevered $P X L$ detector half with 5 sectors. 
Additional details on all three HFT sub-detectors are presented in Section 2, where the most emphasis is placed on the PXL detector that is the first MAPS-based vertex detector at a collider experiment. Section 3 describes the status and initial performance of the HFT detector in the 2014 RHIC run. Section 4 summarizes selected lessons learned from the construction and operation of the PXL detector.

\section{STAR Heavy Flavor Tracker}

\subsection{Silicon Strip Detector}

The outermost layer of the HFT, at the $22 \mathrm{~cm}$ radius, is provided by the SSD detector. The SSD is an existing detector that was used in STAR between 2005 and 2007. The SSD consists of 20 ladders, each with 16 wafers for the total ladder length of $106 \mathrm{~cm}$. Each wafer features 768 strips per side with the strip pitch of $95 \mu \mathrm{m}$ and the stereo angle of $35 \mathrm{mrad}$. The total number of channels in the detector is close to 500k. The detector provides a $20 \mu \mathrm{m}$ resolution in R- $\phi$ and $740 \mu \mathrm{m}$ in $\mathrm{Z}$ (along the beam pipe). The detector is air-cooled, which allows for a low radiation length of approximately $1 \% \mathrm{X}_{0}$. [4].

In order to more closely match HFT requirements, SSD had to be refurbished and its readout electronics had to be upgraded. The readout speed of the detector has been increased from $200 \mathrm{~Hz}$ to $1 \mathrm{kHz}$ by replacing ladder cards and readout boards. The cooling system has also been upgraded to increase the cooling efficiency. The SSD refurbishment was performed at Lawrence Berkeley National Laboratory (LBNL).

\subsection{Intermediate Silicon Tracker}

The Intermediate Silicon Tracker (IST) is located at $14 \mathrm{~cm}$ radius and it is based on singlesided silicon pad sensors with approximately $600 \mu \mathrm{m} \times 6 \mathrm{~mm}$ pitch [5]. The detector is composed of 24 staves, each equipped with 6 silicon pad sensors and 36 APV25-S1 readout chips [6]. The sensitive area of each stave is approximately $50 \mathrm{~cm}$ long. The total number of channels is just above 110k. The detector provides the resolution of $172 \mu \mathrm{m}$ in $\mathrm{R}-\phi$ and $1.8 \mathrm{~mm}$ in Z. The detector is liquid cooled with aluminum cooling tubes integrated into the stave structure. The material budget of this detector is below $1.5 \%$ radiation length. The IST staves were assembled, tested and surveyed at UIC/FNAL and MIT/BNL sites. The readout system of the IST is a copy of the readout system used in the recently completed Forward GEM Tracker (FGT) [7].

\subsection{PXL detector}

The PXL detector, shown in Figure $1 \mathrm{~B}$, consists of two layers of CMOS Monolithic Active Pixel Sensors (MAPS) located at radii of 2.8 and $8 \mathrm{~cm}$. The total of 400 MAPS sensors covers the surface area of $0.16 \mathrm{~m}^{2}$ with $356 \mathrm{M}$ pixels and pixel pitch of $20.7 \mu \mathrm{m}$. The detector features a $185.6 \mu$ s integration time that keeps pileup from consecutive collisions at a manageable level. At the collision rate observed in $\mathrm{Au}+\mathrm{Au}$ collisions $(\sim 50 \mathrm{KHz})$, the $185.6 \mathrm{us}$ integration time limits the occupancy to $\sim 0.03 \%$ in the inner PXL layer. The DCA pointing resolution (separated into the geometrical and Multiple Coulomb Scattering components) of 12 $\oplus 19 \mathrm{GeV} / \mathrm{p} \times \mathrm{c} \mu \mathrm{m}$ for a particle with momentum $\mathrm{p}$ has been achieved due to the high granularity and low material budget with radiation length of $\mathrm{X} / \mathrm{X}_{0}=0.4 \%$ in the inner layer 
$\left(0.5 \% \mathrm{X}_{0}\right.$ in the outer layer) . The low material budget in the PXL detector has been accomplished by combining $50 \mu \mathrm{m}$ thick sensors, aluminum conductor based readout flex cable on the inner layer (copper conductor in the outer layer) and air cooling.

The detector consists of 10 trapezoidal carbon fiber sector tubes, each supporting four 10sensor ladders - one on the inner layer and three on the outer layer. The sectors are assembled into two 5-sector halves and installed on unique, cantilevered mechanical supports that allow for the insertion and retraction of the whole PXL detector from one side of STAR in about 12 hours. The insertion is performed by pushing the detector halves along rails inside a support cylinder and locking them into a reproducible position with kinematic mounts. The mechanical support provides a pixel positional stability at the level of $20 \mu \mathrm{m}$ [8].

The detector has been designed to withstand a moderate radiation environment of the STAR experiment at the level of $90 \mathrm{kRad} /$ year and $2 \times 10^{11}-1 \times 10^{12} 1 \mathrm{MeV} \mathrm{N} \mathrm{eq}_{\mathrm{e}} / \mathrm{cm}^{2}$.

The PXL detector has been designed and built at LBNL and the first copy of the detector (featuring two ladders with aluminum conductor flex cable and 38 ladders with copper conductor cables, as explained in Section 4) was deployed for the 2014 RHIC run. The recently constructed second copy of the detector (with aluminum conductor flex cables in the inner layer) will be used for the 2015 RHIC run. The refurbished 2014 detector can be used as a replacement in case of unexpected damage to the running detector.

\subsubsection{Sensors}

The PXL detector can achieve several of its crucial characteristics, including high spatial resolution, substantial radiation hardness, and low material budget, by employing sensors developed in the MAPS technology.

The sensor chip used in the PXL detector was developed at IPHC, Strasbourg, France, specifically for this detector. The sensor has been fabricated in the $0.35 \mu \mathrm{m}$ technology with a high-resistivity epitaxial layer for improved signal-to-noise performance and increased radiation hardness. The sensor is an array of $928 \times 960$ square pixels with a pitch of $20.7 \mu \mathrm{m}$ that is coupled to column-level discriminators and a zero suppression circuit that provides hit pixel addresses. The pixel array is divided into four sub-arrays to improve compensation of process variations by independent configuration of reference voltages for each sub-array. The pixel array is read out one row at a time in a rolling shutter fashion and the full array processing time defines the sensor integration time, which is equal to $185.6 \mu \mathrm{s}$. The zero suppression system processes one row of data at a time and encodes up to 9 hit clusters per row. The encoded data are then stored in an on-chip memory for intermediate buffering. The memory is structured as two banks of 1800 words each that allow for writing to one bank while reading from another bank. The data are read out by two Low-Voltage Differential Signaling (LVDS) outputs per sensor with a clock speed of $160 \mathrm{MHz}$. [9, 10]. The sensor dissipates approximately 170 $\mathrm{mW} / \mathrm{cm}^{2}$ that allows for operation at room temperature with just air cooling. Many aspects of the sensor operation, including discriminator threshold voltages, can be configured using the built-in Joint Test Action Group (JTAG) protocol.

The measured characteristics of the sensors include an efficiency of $>99 \%$ with an accidental noise hit rate of $<10^{-5}$ even after exposure to an ionizing radiation dose of $150 \mathrm{kRad}$ and non-ionizing radiation dose of $3 \times 10^{12} 1 \mathrm{MeV} \mathrm{N}_{\mathrm{eq}} / \mathrm{cm}^{2}[10,11]$. The single hit spatial 
resolutions of these sensors is $6 \mu \mathrm{m}$ (binary resolution) but can be improved to $3.7 \mu \mathrm{m}$ through cluster centroid reconstruction methods.

\subsubsection{Readout}

The PXL detector readout system follows the mechanical segmentation of the detector into sectors and is divided into 10 parallel identical sections. Signals to and from the sensors are buffered at the end of the ladder, outside of the low mass region, and are carried over $2 \mathrm{~m}$ of fine twisted pair wires (63 $\mu \mathrm{m}$ diameter) to the Mass Termination Board (MTB). The MTB provides additional signal buffering and latch-up protected analog and digital power supplies for each of the four ladders (one sector) that it services. The MTB is connected via approximately $11 \mathrm{~m}$ VHDCI cables to the main FPGA-based readout board (RDO) located in the low radiation area of the STAR experimental hall. The RDO handles configuration of the ladders, receives data from sensors that are read out continuously, and forms sensor data into an event structure in response to triggers from the STAR trigger system. The event data are transferred to the STAR DAQ receiver PCs over a commercially available, high-speed bi-directional fiber link based on Source Interface Unit (SIU) and Readout Receiver Cards (RORC) developed at CERN [12].

The complete PXL detector is composed of 10, fully parallel, sector-MTB-RDO units that are synchronized together by a parallel distribution of the STAR clock and trigger signals and share a common PXL-busy interface. The $10 \mathrm{RDO}$ boards and the clock/trigger/busy distribution board occupy a single $9 \mathrm{U}$ VME crate. The RDO boards were fabricated to conform to the $9 \mathrm{U}$ VME mechanical format for convenience in integration with the existing crates and power supplies at the STAR experiment. The VME bus functionality was not used in the RDO design. Control, configuration and monitoring of the PXL detector are done from a control PC connected to RDO boards via USB.

\section{HFT Status and Performance}

In preparations for taking physics data during the 2014 RHIC run, the SSD and IST detectors were installed inside the STAR TPC inner field cage in the fall of 2013. The SSD detector features a limited number of bad channels: $6 \%$ of dead wafers and $10 \%$ of inactive strips on good wafers. The IST delivers more than $95 \%$ fully functional channels.

The PXL detector was delivered to BNL at the end of 2013 and went through a series of stand-alone tests in the STAR clean room. The detector was installed in the center of the STAR detector in January 2014, shortly before the beginning of the run. The full installation procedure, including insertion and cabling, was accomplished within two days. At the time of installation all 400 sensors of the PXL system were working with less than 2000 bad pixels out of the more than 365 million total. A scan of noise rate versus discriminator threshold was performed and the thresholds were adjusted to give a fake hit rate of $\sim 1.5 \times 10^{-6}$ for each sensor.

Commissioning of the HFT detectors took place mostly in February and March 2014 and included cosmic ray data taking followed by a 4-week run period of $\mathrm{Au}+\mathrm{Au}$ collisions at 14.5 $\mathrm{GeV}$. This time allowed for integration of the readout electronics with the existing STAR DAQ, Trigger, and Slow Controls systems and optimization of the detector performance in the environment with ion collisions. The cosmic ray data collected in absence of magnetic field were used for detection efficiency studies and detector alignment. 
The detection efficiency of the PXL detector was obtained by finding straight cosmic ray tracks in the TPC and with hits in three PXL layers and extrapolating these three hits with a straight-line fit to the fourth PXL layer. Although this study was performed before the detector optimization was complete, the average efficiency over all sensors was determined to be $97.2 \%$.

The same data were also used to align the PXL detector elements. The relative position of all pixels on the sectors and the position of the sectors in the detector half was measured using a coordinate measurement machine during the detector construction phase. The final alignment with cosmic rays took into account the effects of the cooling system airflow and refined the PXL half-to-half alignment. The alignment procedure used cosmic ray tracks found in the TPC and in two layers of the PXL detector half and projected these tracks to the inner and outer layer of the other detector half. The final alignment was achieved by adjusting the position of the sectors to minimize the residuals resulting from comparing hit positions to the track projections. Gaussian fits to the residual (for the inner layer) after alignment reveal $\sigma \leq 25 \mu \mathrm{m}$, which demonstrates the excellent resolution of the PXL detector.

The main part of the physics run was devoted to $200 \mathrm{GeV} \mathrm{Au}+\mathrm{Au}$ collisions and lasted for 17 weeks. During this time, the IST and PXL detectors recorded a total of 1.2 billion minimum bias events while the SSD detector collected 172 million events due to its extended commissioning phase. The detectors were typically triggered at a rate of approximately $1 \mathrm{kHz}$. At this rate the PXL detector operated with a dead time of less than 5\%. The SSD dead time was below $20 \%$ due to the inherent limitation in the old (circa 2000) readout chips.

After completing the preliminary alignment corrections described above, the $200 \mathrm{GeV}$ data were used to estimate the pointing resolution of the PXL detector to the interaction vertex. Figure 2 shows the Distance of Closest Approach (DCA) resolution for tracks found in the TPC which include one IST hit and one hit in each of the two PXL layers as a function of transverse momentum $\mathrm{p}_{\mathrm{T}}$ for protons, pions and kaons. These results prove that the detector meets the design requirement of the pointing resolution $\leq 60 \mu \mathrm{m}$ for kaons with $\mathrm{p}_{\mathrm{T}}=750 \mathrm{MeV} / \mathrm{c}$. A more detailed determination of the alignment corrections is still ongoing and is expected to further improve these results.
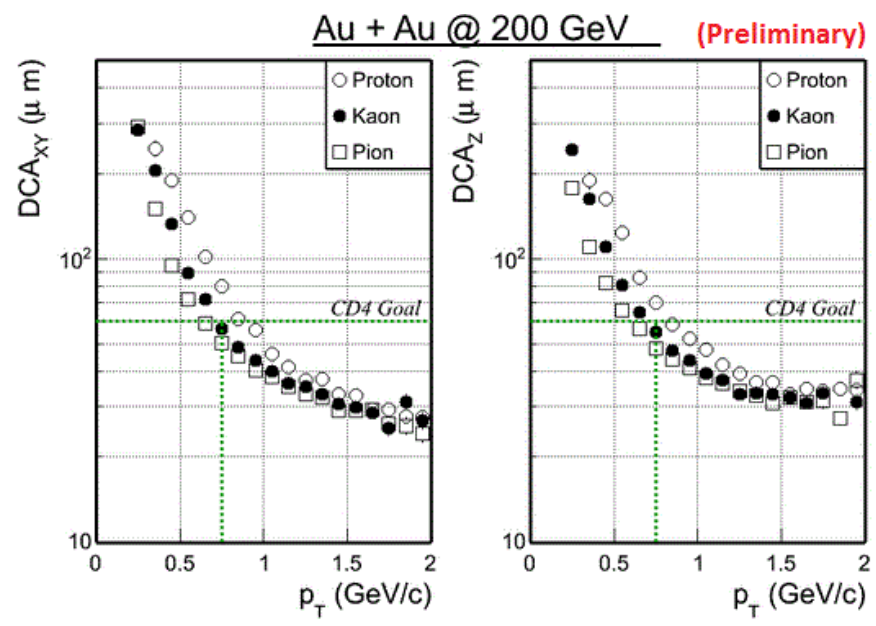

Figure 2 DCA resolution for TPC tracks with one IST hit and hits in both layers of PXL vs. transverse momentum.

During the final part of the run with ${ }^{3} \mathrm{He}+\mathrm{Au}$ collisions SSD and IST recorded data while the PXL detector was only occasionally turned on for further performance studies. 


\section{PXL Detector Lessons Learned}

PXL detector design choices and construction procedures provide a wealth of information that can be useful for future vertex detectors. A brief description of selected lessons learned is presented below.

\subsection{Detector construction}

Efficient assembly of PXL ladders required selecting only high quality sensors that had been diced and thinned to $50 \mu \mathrm{m}$. Development of a probe test setup required the design of a dedicated vacuum chuck that would allow for individual loading of up to 20 thin sensors for automated testing. Testing of thin chips, which typically curve at this reduced thickness, proved to be challenging and the default probe pin design delivered by the probe card vendor had to be optimized to allow for additional overdrive distance to improve contact reliability with a nonflat surface. The changes were the increase in beam length of the probes along with the probe taper which had the functional effect of reducing the spring constant of the probe and giving longer travel for equivalent probe force. The probe card was equipped with readout electronics necessary to perform full sensor characterization, including noise and operating threshold measurements, as well as the full speed readout at $160 \mathrm{MHz}$. The testing functionality built into the sensors was crucial at this stage. The overall yield of the detector grade sensors during the PXL detector production varied between 46 and $60 \%$.

The construction of PXL ladders relied on custom made vacuum fixtures that provided high precision alignment of individual ladder components. The sensors were manually placed, butted together, and glued to the readout flex cable using a double-sided adhesive tape, preventing damages arising from different thermal expansion characteristics. Sensor alignment on the ladder was significantly enhanced by the use of Deep Reactive Ion Etching (DRIE) during wafer production to create dicing trenches. Sensors were connected to the flex readout cable via standard wire bonding and the wires were encapsulated for protection.

Production of the double-sided aluminum flex cables encountered several technical difficulties and was delayed. For this reason, the first PXL detector constructed featured only two inner ladders equipped with aluminum-conductor flex cables. The remaining 38 ladders were assembled using a copper-conductor backup alternative. The use of copper instead of aluminum effectively doubles the material budget of the PXL flex cable and translates to an increase in the ladder radiation length from approximately $0.4 \%$ to $0.5 \%$.

\subsection{Engineering run}

A crucial milestone in the PXL detector development was the engineering run performed with a prototype detector inserted into STAR for several weeks of the 2013 RHIC run. The prototype detector featured full mechanical support structure and was equipped with three sectors and the associated readout electronics. The initial production of ladders for the engineering run detector revealed several issues that lead to short circuits in the assembled ladders. The issues were resolved by extending the adhesive tape layer beyond the sensor footprint and by adding a solder mask layer to the flex cable corresponding to $0.0075 \%$ radiation length (this layer was initially removed to minimize the material budget of the assembly). 
The assembly of sectors for the engineering run revealed a mechanical interference between driver boards located at the end of inner ladders in neighboring sectors. As a consequence, the engineering run detector featured full sectors separated by at least one empty sector (a sector tube without any ladders attached). Ultimately, the geometry of the sector tube required modifications and the radius of the inner layer increased from the original $2.5 \mathrm{~cm}$ to 2.8 $\mathrm{cm}$. The engineering run experience led also to optimization of the MTB. The new board features remote monitoring of the individual ladder current consumption (analog and digital) and remote control of the over-current protection threshold and the ladder power supply voltage.

\subsection{Radiation related damage observed in the 2014 RHIC run}

During the commissioning phase of the PXL detector with $14.5 \mathrm{GeV} \mathrm{Au}+\mathrm{Au}$ collisions, the detector started to exhibit performance changes that indicated radiation-related sensor damage. The damage continued to accumulate into the first two weeks of the $200 \mathrm{GeV} \mathrm{Au}+\mathrm{Au}$ run until a set of operational methods was applied, which effectively limited further damage to the sensors. The observed damage appeared to be latch-up related and took on many different forms: increased digital current consumption, damage to pixel columns, loss of full or partial pixel subarrays, etc. Most of the damage occurred in sensors in the inner layer, but even some of the sensors in the outer layer were affected. A total of 16 of the 400 sensors in the PXL detector were damaged, which corresponds to a loss of $14 \%$ of the active surface on the inner layer and $1 \%$ on the outer layer. This limited damage did not interfere with the successful completion of the 2014 physics run.

In the set of operational methods applied to limit the observed sensor damage, the most important modification was the reduction of the over-current protection threshold. The initial threshold of $400 \mathrm{~mA}$ above the measured operating digital current on each ladder was decreased to $120 \mathrm{~mA}$. In addition, the PXL detector was only turned on for data taking when the collision rate was below a certain value and all the sensors were power-cycled and reconfigured every 15 minutes. With all these procedures in place, only two more sensors exhibited additional damage in the remaining three months of the run. The PXL detector that will be installed for the 2015 RHIC run will operate with all these methods applied from the beginning of the run. We expect that this approach will prevent future sensor damage in the PXL detector.

The failure mechanism is now under extensive investigation by exposing existing PXL ladders and thinned sensors to heavy ion beam irradiation. This test is mainly aimed at characterizing the damage mechanism and at defining a safe operation envelope of latch-up current threshold settings for the 2015 run.

\section{Summary and Outlook}

The new vertex detector, the Heavy Flavor Tracker, composed of three silicon detector subsystems was successfully integrated in the STAR experiment and commissioned for the 2014 $\mathrm{Au}+\mathrm{Au}$ RHIC run. The HFT detector recorded approximately 1.2 billion minimum bias $\mathrm{Au}+\mathrm{Au}$ collision events and preliminary results indicate that the DCA pointing resolution performance of the HFT meets or exceeds the design goals. The HFT will significantly enhance heavy flavor measurement capabilities of the STAR experiment by allowing for reconstruction of displaced decay vertices very close to the interaction point. 
The innermost subsystem of the HFT, the PXL detector, is the first tracking detector in a collider experiment that is based on CMOS sensors. At the beginning of the run, the PXL detector performance indicated sensor damage that was related to the radiation environment in STAR but later appeared to be significantly suppressed by implementing operational methods. A second PXL detector equipped with aluminum flex cables on the inner ladders was constructed in the summer 2014 and will be installed in STAR for the 2015 RHIC run. The ladders damaged during the 2014 run were replaced and the refurbished detector was shipped to STAR to serve as a hot spare.

The PXL detector demonstrates that the employed state-of-the-art MAPS technology is suitable for vertex detectors, and as such it leads the way for future vertex detectors based on this technology, for example in the ALICE ITS upgrade or the CBM experiment.

\section{Acknowledgments}

This work was supported by the Director, Office of Science, Office of Nuclear Science of the U.S. Department of Energy under Contract No. DE-AC02-05CH11231.

We gratefully acknowledge the PICSEL group of IPHC Strasbourg (Marc Winter et al.) for the development of the PXL detector sensors.

\section{References}

[1] J. Adams et al., Experimental and theoretical challenges in the search for the quark-gluon plasma: The STAR Collaboration's critical assessment of the evidence from RHIC collisions, Nucl. Phys. A 757 (2005), 102-183.

[2] C. Chasman et al., A Heavy Flavor Tracker for STAR, (2008), LBNL/PUB-5509-2008B.

[3] M.Winter, Achievements and perspectives of CMOS pixel sensors for charged particle tracking, Nucl. Instrum. Meth. A 623 (2010), 192-194

[4] L. Arnold, The STAR silicon strip detector (SSD), Nucl. Instrum. Meth. A 499 (2003), 652-658

[5] Y. Wang, Intermediate Silicon Tracker For STAR HFT Upgrade, QuarkMatter 2014

[6] M.J. French, et al. Design and results from the APV25, a deep sub-micron CMOS front-end chip for the CMS tracker, Nucl. Instrum. Meth. A, 466 (2001), p. 359

[7] G. Visser et al. A Readout System Utilizing the APV25 ASIC for the Forward GEM Tracker in STAR, IEEE Real Time Conference Record, Berkeley, CA, 2012

[8] L. Greiner et al., A MAPS based vertex detector for the STAR experiment at RHIC, Nucl. Instrum. Meth. A, 650 (2011), 68-72

[9] C. Hu-Guo et al., First reticule size MAPS with digital output and integrated zero suppression for the EUDET-JRA1 beam telescope, Nucl. Instrum. Meth. A 623 (2010), 480-482

[10] A. Dorokhov et al., High-resistivity CMOS pixel sensors and their application to the STAR PIXEL detector, Nucl. Instrum. Meth. A 650 (2011), 174-177

[11] Jérôme Baudot, et al. Optimization of CMOS pixel sensors for high performance vertexing and tracking, Nucl. Instrum. Meth. A 732 (2013), 480-483, doi: 10.1016/j.nima.2013.06.101

[12] http://alice-proj-ddl.web.cern.ch/alice-proj-ddl/ 